\title{
CONSUMO DE ÁLCOOL ENTRE OS TRABALHADORES DO CORTE DA CANA-DE-AÇÚCAR - prevalência e fatores associados
}

\author{
Jorge Augusto Beck Filho*, Andréa Monteiro Amorim**, Helena Fraga Maia* \\ Autor correspondente: Jorge Augusto Beck Filho - jorgebeckfilho@gmail.com \\ *Universidade do Estado da Bahia. Departamento Ciências da Vida I \\ **Faculdade de Tecnologia e Ciências. Mestrado Profissional em Bioenergia
}

\begin{abstract}
Resumo
Objetivo: Estimou-se a prevalência e os fatores associados ao consumo de álcool entre os trabalhadores do corte da cana-de-açúcar por meio de um estudo de corte transversal com canavieiros do município de Amélia Rodrigues, Bahia. Material e Métodos: Dados primários foram coletados com aplicação de formulários. Para identificar uso abusivo de álcool, foi utilizado o Test - AUDIT. A magnitude da associação entre as variáveis estudadas e o uso abusivo de bebidas alcoólicas foi estimada pela razão de chances (odds ratio, OR), adotando-se o intervalo de confiança a 95\% como medida de precisão. Posteriormente, foram realizadas análises multivariadas utilizando-se a regressão logística. Resultados: Dos 180 indivíduos selecionados 50,0\% apresentaram AUDIT $\geq 8$, caracterizando consumo abusivo de álcool. Este se mostrou associado com sentimento de tristeza ao final da jornada de trabalho, insatisfação com o trabalho e com baixa qualidade de vida, mesmo após ajuste por variáveis sociodemográficas, ocupacionais e relativas a acidentes. Conclusão: A prevalência de consumo abusivo de álcool entre os canavieiros é elevada e os principais fatores associados ao consumo são a insatisfação com o trabalho, o sentimento de tristeza ao final da jornada de trabalho e a baixa qualidade de vida.
\end{abstract}

Palavras-chave: Alcoolismo; Condições de Trabalho; Trabalhador Rural; Saúde; Ambiente. 


\title{
ALCOHOL CONSUMPTION AMONG SUGARCANE CUTTERS \\ - prevalence and associated factors
}

\begin{abstract}
Objective: The prevalence of alcohol consumption and associated factors among sugarcane workers was estimated by means of a cross-sectional study in the municipality of Amelia Rodrigues, Bahia. Methods: Primary data was collected through the application of forms. In order to identify alcohol abuse, the AUDIT Test was employed. The magnitude of the association among the studied variables and the abusive use of alcoholic beverages were estimated using the odds ratio (OR), adopting $95 \%$ as a measure of precision. Afterwards, multivariate analyses were performed using logistic regression. Out of the 180 individuals selected, 50.0\% presented AUDIT $\geq 8$, characterizing alcohol consumption at levels considered as abusive. Results: This factor was associated to a feeling of sadness at the end of the working day, satisfaction with the work, and with low quality of life, even after adjustments for socio-demographic and occupational variables and those related to accidents. Conclusion: Prevalence of alcohol abuse among sugarcane cutters is high and the main factors associated to the consumption are dissatisfaction with work, the feeling of sadness at the end of the working day and low quality of life.
\end{abstract}

Keywords: Alcoholism; Working Condition; Rural work; Health; Environment.

\section{INTRODUÇÃO}

O álcool é uma substância psicoativa legal e socialmente aceita que causa a dependência conhecida como alcoolismo. ${ }^{(1,2)}$ Reconhecido como doença pela Organização Mundial de Saúde (OMS) desde 1948, o alcoolismo representa um dos problemas socioeconômicos mais graves para a saúde mundial.(2) A explicação para a disseminação dessa patologia associa-se à facilidade com que esta substância pode ser adquirida, a sua aceitação social e a adaptação à maioria das culturas. ${ }^{(1)}$ Estima-se que a dependência deva atingir entre 10\% a 15\% da população mundial, sendo o impacto danoso considerado como responsável por $8 \%$ a $14,9 \%$ do total de problemas de saúde dos países em desenvolvimento, entre eles o Brasil. ${ }^{(2)}$
O consumo excessivo de bebidas alcoólicas ocasiona, além de problemas econômicos, transtornos sociais e psicológicos, ao que se atribui a relevância do seu estudo e das possíveis causas. ${ }^{(3)}$ A dependência envolve aspectos comportamentais, cognitivos e fisiológicos que se desenvolvem após o uso repetido. Nesse sentido, o consumo do álcool está associado a uma ampla variedade de doenças e problemas de ordem social e jurídica. A prática tem causado riscos substanciais e, eventualmente, danos para o indivíduo, entre os quais se incluem intoxicações, dependência física ou psíquica, além de, muitas vezes, afetar as suas relações sociais e laborais, representando um imenso problema para muitas sociedades em todo o mundo. ${ }^{(3,4)}$ 
Nas situações de trabalho, são considerados fatores de risco para o consumo do álcool as atividades socialmente desprivilegiadas e/ou onde a tensão é constante. Isto ocorre como um mecanismo de defesa, uma fuga do sofrimento mental, da sobrecarga emocional e mesmo das condições de trabalho, tornando o consumo do álcool uma "válvula de escape", um aliado para o alívio da tensão, do desprazer e do sofrimento, induzindo a quadros graves de dependência alcoólica. ${ }^{(5)}$ Os agentes estressores ligados ao trabalho têm origens diversas, como as condições externas, a economia, a política e as exigências culturais, embora a mais importante fonte de tensão seja a condição interior de cada trabalhador. ${ }^{(6,7)}$ Sabe-se também que o aumento dos desgastes físico, social e mental pode contribuir para a elevação do consumo de álcool entre os trabalhadores, inclusive os da cultura canavieira. Todavia, estudos sobre tal temática para esta específica população pouco são realizados e, por conseguinte, políticas públicas locais de controle deixam de ser desenvolvidas. Desta forma, este trabalho propõe-se a estudar a prevalência e os fatores associados ao consumo de álcool entre os trabalhadores do corte da cana-de-açúcar, do município de Amélia Rodrigues, Bahia.

\section{MATERIAL E MÉTODOS}

Trata-se se um estudo transversal de base institucional realizado com trabalhadores do corte da cana-de-açúcar do município de Amélia Rodrigues, Bahia. Foram convidados a participar do inquérito todos os residentes no referido município e que fossem filiados ao Sindicato dos Trabalhadores e Trabalhadoras Rurais de Amélia Rodrigues. Foram excluídos aqueles que estavam afastados, temporária ou definitivamente, por acidentes ou doenças ocupacionais. Para estimar a prevalência de consumo abusivo de álcool, tomou-se por base a frequência de $17,9 \%$ relatada por Gonçalves e Villar Luís, ${ }^{(8)}$ no município de Ribeirão Preto, SP. Considerou-se o nível de confiança de $95 \%$, para uma população de 500 trabalhadores, com frequência esperada do evento de $25,0 \%$ e o pior resultado aceitável de $18,0 \%$. Com tais parâmetros, o tamanho da amostra foi estimado em 114 indivíduos. Em função de possíveis perdas de informações durante o período da coleta de dados, este número foi aumentado para 180 trabalhadores, procurando-se atingir o nível de significância de 99\%. O cálculo do tamanho amostral foi realizado com a calculadora Winpepi (http://www.brixtonhealth.com/pepi4windows.html).

Dados primários foram coletados por meio da aplicação de formulários. A coleta de dados foi realizada no mês de março de 2014, nas dependências de uma usina local, e conduzida pelo pesquisador principal e por estudantes previamente treinados para tal. Um instrumento estruturado foi desenvolvido pelos autores do estudo e continha blocos de questões sobre variáveis socioeconômicas, demográficas, ocupacionais e relativas à saúde dos trabalhadores envolvidos no corte de cana-de-açúcar. Com o objetivo de identificar possíveis dependentes de álcool, foi utilizado o Alcohol Use Disorders Identification Test - AUDIT, instrumento elaborado pela Organização Mundial de Saúde (OMS), em 1992, traduzido e validado no Brasil em 2001. (9) Desenvolvido pela OMS como instrumento de rastreamento para uso problemático de álcool foi idealizado para classificar o nível de dependência. Utilizou-se como ponto de corte a classificação $\geq 8$ pontos para aqueles que bebiam de modo abusivo ou que apresentavam consumo de risco de acordo com evidencias científicas recentes. ${ }^{(10)}$

Entre as variáveis sociodemográficas, questionou-se o sexo, a idade em anos completos e a cor da pele, definida pelo entrevistador e categorizada conforme o IBGE, em (http://www.ibge.gov. br/home/estatistica/populacao/condicaodevida/ indicadoresminimos/conceitos.shtm) e colapsadas em pretos e pardos. A escolaridade foi definida em analfabeto ou $1^{\circ}$ grau incompleto e/ou $1^{\circ}$ grau completo ou maior. A situação conjugal foi categorizada em casados ou vivendo em união estável e vivendo sem companheiro. A renda mensal foi definida em menor ou igual a um salário mínimo ou 
maior que um salário mínimo. Já a crença religiosa foi definida em ter ou não ter.

Entre as características ocupacionais coletadas, o tempo de trabalho no corte da cana-de-açúcar, foi caracterizado em anos e o tempo de trabalho diário no corte, em horas. A satisfação com o trabalho foi categorizada em sim e não, assim como o sentimento de tristeza ao final da jornada. Investigou-se ainda, como o trabalhador avaliava sua qualidade de vida e esta foi definida em boa, ruim ou muito ruim. Quanto aos acidentes, foram investigadas a realização de treinamento para desenvolvimento da atividade, a ocorrência de acidentes de trabalho, se o trabalhador sabia o que são Equipamentos de Proteção Individual (EPI) e se considerava que os EPI disponibilizados estavam adequados. Todas essas variáveis foram consideradas como dicotômicas. Finalmente, investigou-se o grau de perigo no trabalho por meio do emprego de uma escala visual analógica graduada de zero a dez e posteriormente categorizado em $\leq 5$ ou $>5$.

O banco de dados foi confeccionado no programa Excel for Windows e a digitação foi realizada em dupla, com o objetivo de minimizar erros de preenchimento. Na sequência, foram realizados procedimentos para limpeza do mesmo. Análises bivariadas foram realizadas com o intuito de identificar o conjunto e variáveis que mais se associaram com o uso abusivo de bebidas alcoólicas. A magnitude da associação entre as variáveis estudadas e o uso abusivo de bebidas alcoólicas foi estima- da pelo cálculo da razão de chances (odds ratio, OR), adotando-se o intervalo de confiança a 95\% (IC95\%) como medida de precisão. Posteriormente, foram realizadas análises multivariadas utilizando-se a regressão logística. Compuseram o modelo final as variáveis que mostraram níveis de significância estatística na análise bivariada, segundo um $p<0,20$. Os pacotes estatísticos utilizados foram o Excel for Windows (versão 3.2) e o Stata (versão 12.0). O projeto de pesquisa foi aprovado pela CEP-Protocolo n ${ }^{\circ} 484.643 / 09 / 12 / 2013$.

\section{RESULTADOS}

Dentre os 180 cortadores de cana-de-açúcar convidados a participar do inquérito, 90 (50,0\%) exibiram AUDIT $\geq 8$ pontos sendo portanto identificados como consumidores de bebidas alcoólicas em níveis abusivos ou de risco. Na tabela 1 encontram-se descritos as frequências e as análises bivariadas e multivariadas dos dados sociodemográficos e o uso abusivo de bebidas alcoólicas. De modo geral, as análises bivariadas não mostraram significância estatística, exceto para a associação entre crença religiosa e uso abusivo de bebidas alcoólicas $(p=0,048)$. O sexo, embora não tenha apresentado associação estatisticamente significante o uso abusivo de bebidas alcoólicas $(p<0,20)$, foi considerado nas análises subsequentes para fins de ajustes do modelo multivariado (Tabela 1). 
Tabela 1 - Prevalência (n/\%) e Razões de chances (Odds ratio) do uso abusivo de bebidas alcoólicas (AUDIT · 8) por variáveis sociodemográficas de trabalhadores do corte da cana-de-açúcar, Amélia Rodrigues, Bahia, 2014. N=180

\begin{tabular}{|c|c|c|c|c|}
\hline \multirow[b]{2}{*}{ Variáveis } & \multicolumn{3}{|c|}{ Uso abusivo de bebidas alcoólicas (AUDIT $\geq 8$ ) } & \multirow[b]{2}{*}{ Valor dep } \\
\hline & $\mathrm{n}(\%)$ & $\begin{array}{l}\text { OR bruta } \\
\text { (IC 95\%) }\end{array}$ & $\begin{array}{c}\text { OR aj ustada } \\
\text { (I C } 95 \% \text { ) }\end{array}$ & \\
\hline \multicolumn{5}{|l|}{ Sexo } \\
\hline Feminino & $47(26,1)$ & 1 & 1 & \\
\hline Masculino & $133(73,9)$ & $1,90(0,96-3,75)$ & $1,55(0,75-3,18)$ & 0,228 \\
\hline \multicolumn{5}{|l|}{ Idade } \\
\hline $40 \mathrm{e}+$ & $110(61,1)$ & 1 & 1 & \\
\hline 18 a 39 & $70(38,9)$ & $1,09(0,60-1,99)$ & $1,21(0,61-2,38)$ & 0,581 \\
\hline \multicolumn{5}{|l|}{ Cor da pele } \\
\hline Brancos & $16(8,9)$ & 1 & 1 & \\
\hline Pretos e pardos & $164(91,1)$ & $1,75(0,60-5,03)$ & $1,77(0,54-5,79)$ & 0,343 \\
\hline \multicolumn{5}{|l|}{ Situação conj ugal } \\
\hline Casados ou vivendo em uni ão estável & $114(63,3)$ & 1 & 1 & \\
\hline Vivendo sem companheiro & $66(36,7)$ & $1,21(0,65-2,22)$ & $0,98(0,51-1,90)$ & 0,970 \\
\hline \multicolumn{5}{|l|}{ Escolaridade } \\
\hline $1^{\circ}$ grau completo ou maior & $36(20,0)$ & 1 & 1 & \\
\hline Analfabeto ou $1^{\circ}$ grau incompleto & $114(80,0)$ & $2,36(1,10-5,08)$ & $2,17(0,93-5,04)$ & 0,078 \\
\hline \multicolumn{5}{|l|}{ Renda mensal (em salários mínimos) } \\
\hline$>1$ & $37(20,6)$ & 1 & 1 & \\
\hline$\leq 1$ & $143(79,4)$ & $1,61(0,77-3,36)$ & $1,36(0,62-2,95)$ & 0,435 \\
\hline \multicolumn{5}{|l|}{ Crença } \\
\hline Ter & $157(87,2)$ & 1 & 1 & \\
\hline Não ter & $23(12,8)$ & $3,26(1,22-8,70)$ & $2,84(1,01-8,05)$ & 0,048 \\
\hline
\end{tabular}

* Variáveis com valor de p· 0,20 integraram o modelo da regressão logística multivariada, ajustado por sexo, grau de instrução e crença.

O uso abusivo de bebidas alcoólicas associou-se significativamente à variável "satisfação com o trabalho" (OR = 3,72; IC95\%: 1,53-9,07), ao "sentimento de tristeza ao final da jornada de trabalho"
(OR = 3,17; IC95\%: 1,25-8,03) e com a avaliação pessoal da "qualidade de vida" (OR = 9,52; IC $95 \%$ : $3,67-24,69$ ), (tabela 2). 
Tabela 2 - Prevalência (n/\%) e Razões de chances (Odds ratio) do uso abusivo de bebidas alcoólicas (AUDIT · 8) por variáveis ocupacionais de trabalhadores do corte da cana-de-açúcar, Amélia Rodrigues, Bahia, 2014. N=180

\begin{tabular}{|c|c|c|c|c|}
\hline \multirow[b]{2}{*}{ Var iáveis } & \multicolumn{3}{|c|}{ Uso abusivo de bebidas alcoólicas (AUDIT $\geq 8$ ) } & \multirow[b]{2}{*}{ Valor dep } \\
\hline & $\mathbf{n}(\%)$ & $\begin{array}{l}\text { ORbruta } \\
\text { (IC 95\%) }\end{array}$ & $\begin{array}{l}\text { OR ajustada } \\
\text { (IC } 95 \% \text { ) }\end{array}$ & \\
\hline \multicolumn{5}{|c|}{$\begin{array}{l}\text { Tempo de tr abalho no corte da cana-de-açúcar } \\
\text { (anos) }\end{array}$} \\
\hline$<10$ & $79(43,9)$ & 1 & 1 & \\
\hline$\geq 10$ & $101(56,1)$ & $0,60(0,33-1,10)$ & $0,88(0,36-2,10)$ & 0,774 \\
\hline \multicolumn{5}{|c|}{ Tempo de tr abal ho diár io no corte(hor as) } \\
\hline$\leq 8$ & $144(80,0)$ & 1 & 1 & \\
\hline$>8$ & $36(20,0)$ & $0,75(0,36-1,57)$ & $0,56(0,19-1,61)$ & 0,286 \\
\hline \multicolumn{5}{|l|}{ Satisfação com o trabalho } \\
\hline Satisfeito & $71(39,4)$ & 1 & 1 & \\
\hline Insatiff eito & $109(60,6)$ & $6,44(3,27-12,6)$ & $3,72(1,53-9,07)$ & 0,004 \\
\hline \multicolumn{5}{|c|}{$\begin{array}{l}\text { Sentimento de cansaço ao final da jornada de } \\
\text { trabalho }\end{array}$} \\
\hline Não & $22(12,2)$ & 1 & 1 & \\
\hline $\operatorname{Sim}$ & $158(87,8)$ & $1,23(0,50-3,01)$ & $0,78(0,24-2,49)$ & 0,679 \\
\hline \multicolumn{5}{|c|}{$\begin{array}{l}\text { Sentimento de tr isteza ao final da jor nada de } \\
\text { trabalho }\end{array}$} \\
\hline Não & $64(35,6)$ & 1 & 1 & \\
\hline Sim & $116(64,4)$ & $6,78(3,35-13,74)$ & $3,17(1,25-8,03)$ & 0,015 \\
\hline \multicolumn{5}{|l|}{ Qualidade de vida refer ida } \\
\hline Boa & $52(28,9)$ & 1 & 1 & \\
\hline Ruim / m uito ruim & $128(71,1)$ & $9,80(4,25-22,60)$ & $9,52(3,67-24,69)$ & $<0,001$ \\
\hline
\end{tabular}

* Variáveis com valor de p· 0,20 integraram o modelo da regressão logística multivariada, ajustado por sexo, grau de instrução, crença, satisfação com o trabalho e sentimento de tristeza ao final da jornada de trabalho.

$\mathrm{Na}$ tabela 3 descrevem-se as frequências, as razões de chance bruta e ajustada entre o uso abusivo de bebidas alcoólicas e variáveis relacionadas com acidentes de trabalho. Se associaram de modo significativo com o uso abusivo de bebidas alcoólicas as variáveis "sabe o que são equipamentos de proteção individual (EPI)" ( $p=0,166)$ e "se considera que os EPI disponibilizados são adequados" $(p=0,153)$, (tabela 3$)$. 
Tabela 3 - Prevalência ( $\mathrm{n} / \%$ ) e Razões de chances (Odds ratio) do uso abusivo de bebidas alcoólicas (AUDIT · 8) por variáveis relacionadas com acidentes para trabalhadores do corte da cana-de-açúcar, Amélia Rodrigues, Bahia, 2014. $\mathrm{N}=180$

\begin{tabular}{|c|c|c|c|c|}
\hline \multirow[b]{2}{*}{ Variáveis } & \multicolumn{3}{|c|}{ Uso abusivo de bebidas al codilicas (AUDI $T \geq 8$ ) } & \multirow[b]{2}{*}{ Valor de $\mathrm{p}$} \\
\hline & $n(\%)$ & $\begin{array}{l}\text { OR bruta } \\
\text { (IC } 95 \%)\end{array}$ & $\begin{array}{l}\text { OR aj ustada } \\
\text { (IC } 95 \% \text { ) }\end{array}$ & \\
\hline $\begin{array}{l}\text { Realizaçáo de } \text { treinamento } \\
\text { desenvol vimento da ativi dade de trabal ho }\end{array}$ & & & & \\
\hline Não & $105(58,3)$ & 1 & 1 & \\
\hline $\operatorname{Sim}$ & $75(41,7)$ & $0,66(0,36-1,20)$ & $0,90(0,37-2,16)$ & 0,818 \\
\hline \multicolumn{5}{|l|}{ Grau de perigo no trabalho (0 a 10) } \\
\hline$\leq 5$ & $26(14,4)$ & 1 & 1 & \\
\hline$>5$ & $154(85,6)$ & $5,17(1,85-14,43)$ & $2,08(0,52-8,33)$ & 0,299 \\
\hline \multicolumn{5}{|l|}{ Acidentes de trabalho œcorrem com frequência } \\
\hline Não & $47(26,1)$ & 1 & 1 & \\
\hline $\operatorname{Sim}$ & $133(73,9)$ & $1,68(0,85-3,31)$ & $1,18(0,42-3,30)$ & 0,743 \\
\hline \multicolumn{5}{|l|}{ Sofreu aci dente de trabalho } \\
\hline Não & $101(56,1)$ & 1 & 1 & \\
\hline $\mathrm{Sim}$ & $79(43,9)$ & $1,50(0,83-2,71)$ & $1,06(0,41-2,70)$ & 0,902 \\
\hline \multicolumn{5}{|l|}{ Sabe o queé EPI } \\
\hline $\mathrm{Sim}$ & $167(92,8)$ & 1 & 1 & \\
\hline Não & $13(7,2)$ & $6,12(1,31-28,49)$ & $3,40(0,59-19,71)$ & 0,166 \\
\hline \multicolumn{5}{|l|}{$\begin{array}{l}\text { Considera os EPI que são di sponibilizados camo } \\
\text { adequados }\end{array}$} \\
\hline Não & $106(58,9)$ & 1 & 1 & \\
\hline $\mathrm{Sim}$ & $74(41,1)$ & $0,35(0,19-0,65)$ & $0,53(0,22-1,26)$ & 0,153 \\
\hline
\end{tabular}

* Variáveis com valor de p· 0,20 integraram o modelo da regressão logística multivariada, ajustado por sexo, grau de instrução, crença, satisfação com o trabalho e sentimento de tristeza ao final da jornada de trabalho, saber o que é EPI, considera EPI adequado.

Na Tabela 4 apresentam-se as variáveis do modelo final de regressão logística. Na análise multivariada, o uso abusivo de bebidas alcoólicas se mostrou associado com o sentimento de tristeza ao final da jornada de trabalho $(\mathrm{OR}=3,12$; IC $95 \%$ : $1,18-7,58)$, com a satisfação com o trabalho (OR
$=3,64 ;$ IC95\%: $1,53-8,68)$, e com a avaliação da sua qualidade de vida (OR = 9,07; IC95\%: $3,54 \%$ - 23,21), mesmo após ajuste por demais variáveis sociodemográficas, ocupacionais e relativas a acidentes de trabalho. (Tabela 4).

Tabela 4 - Odds ratios bruto e ajustado para uso abusivo de bebidas alcoólicas (AUDIT · 8) para trabalhadores do corte da cana-de-açúcar, segundo as variáveis que permaneceram no modelo da regressão logística multivariada. Amélia Rodrigues, Bahia, 2014. N=180

\begin{tabular}{|c|c|c|c|}
\hline \multirow{2}{*}{ Variável } & \multicolumn{2}{|c|}{ Uso abusivo de bebidas alcólicas (AUDIT $\geq 8$ ) } & \multirow[b]{2}{*}{ Valor de $p$} \\
\hline & $\begin{array}{l}\text { OR bruto } \\
\text { (IC 95\%) }\end{array}$ & $\begin{array}{l}\text { OR ajustado } \\
\text { (IC } 95 \% \text { ) }\end{array}$ & \\
\hline Sentimento de tristeza ao final da jornada de trabalho & $6,78(3,35-13,74)$ & $3,12(1,28-7,58)$ & 0,012 \\
\hline Satisfação com otr abalho & $6,44(3,27-12,6)$ & $3,64(1,53-8,68)$ & 0,003 \\
\hline Qualidade de vida r ferida & $9,80(4,25-22,60)$ & $9,07(3,54-23,21)$ & $<0,0001$ \\
\hline
\end{tabular}

\footnotetext{
* Variáveis com valor de p· 0,20 integraram o modelo da regressão logística multivariada, ajustado por sexo, grau de instrução, crença, satisfação com o trabalho e sentimento de tristeza ao final da jornada de trabalho, saber o que é EPI, considera EPI adequado.
} 


\section{DISCUSSÃO}

A prevalência para o consumo abusivo de bebidas alcoólicas em trabalhadores do corte da cana-de-açúcar do município de Amélia Rodrigues foi considerada elevada $(50,0 \%)$. Evidências de consumo de risco nesta população também foram relatadas por Gonçalves e Villar Luís. ${ }^{(8)}$ De acordo com estas autoras, 34,5\% dos canavieiros de Ribeirão Preto, São Paulo, referiram beber quantidades elevadas de álcool em curtos períodos de tempo, obtendo, deste modo, pontuação elevada no instrumento AUDIT. Todavia, $17,9 \%$ deles foram classificados como tendo padrão de consumo do tipo binge. De acordo com o National Institute on Alcohol Abuse and Alcoholism (NIAAA), binge drinking refere-se ao uso pesado episódico de álcool e é definido como o consumo de cinco ou mais doses alcoólicas por homens ou de quatro ou mais doses por dentro de um período de duas horas.

O presente estudo revelou que a insatisfação com o trabalho do corte da cana associou-se positivamente com o consumo abusivo de álcool, assemelhando-se aos resultados apresentados por Gonçalves e Villar Luís, ${ }^{(8)}$ Amato, ${ }^{(10)}$ e Ferreira. ${ }^{(11)}$ Estes pesquisadores salientaram que a insatisfação no trabalho, principalmente com os baixos salários, as tensões vivenciadas no campo e o desenvolvimento das atividades a todo tempo de forma controlada e vigiada, aparecem como fator predisponente para o desestímulo, tornando esta classe de trabalhadores vulnerável ao consumo de álcool.

Ainda com relação à insatisfação com o trabaIho como fator de risco para a saúde, Fehlberg, ${ }^{(12)}$ em Pelotas, Rio Grande do Sul, identificaram um risco elevado para a ocorrência de acidentes de trabalho (OR = 2,77; IC95\%: 1,38 - 5,57) entre aqueles que referiam insatisfação com o trabalho realizado. Ao investigarem a ocorrência destes eventos em ambiente laboral e sua associação com fatores de risco, as autoras concluíram que a possibilidade de redução da carga de trabalho, sem o correspondente ônus na produção, e a satisfação decorrente da realização de uma atividade socialmente valo- rizada, possivelmente resultariam em diminuição da prevalência de acidentes entre trabalhadores rurais. Como no presente estudo a associação entre a insatisfação com o trabalho e o uso abusivo de bebidas alcoólicas teve magnitude elevada, pode-se inferir risco elevado para acidentes de trabalho entre aqueles que cortam cana no município de Amélia Rodrigues. Deste modo, ações de vigilância em saúde e inspeções das Delegacias Regionais do Trabalho devem ser periódicas e efetivas.

O grau de satisfação e motivação pelo trabalho são influenciados por eventuais desajustes entre as expectativas do trabalhador e o que é vivenciado na empresa. Tais condições propiciam o surgimento de atitudes que podem abalar a harmonia e a estabilidade psicológica dentro do ambiente laboral reforçando o descontentamento para a realização das atividades laborais. ${ }^{(8,13)}$ Dito de outra forma, a insatisfação resulta da complicada interação entre as condições gerais de vida, as relações e o processo de trabalho, ${ }^{(14)}$ o que parece estar associado com as variáveis investigadas no presente estudo. O resultado obtido pode ser parcialmente atribuído ao fato de que os cortadores da cana-de-açúcar vivem em condições de trabalho desfavoráveis, com carga excessiva de trabalho, associada à baixa remuneração, que possibilita a origem de efeitos adversos à saúde física e mental, estabelecendo-se como um fator de risco para o consumo de drogas, dentre elas o álcool.

Sentimento de tristeza mostrou-se, no presente estudo, relacionado ao uso excessivo de bebida alcoólica. Dados do Ministério da Saúde ${ }^{(2)}$ apontam que $17 \%$ dos trabalhadores brasileiros no auge da vida profissional, na faixa etária entre e 25 e 40 anos, desenvolvem a depressão, caracterizada por tristeza excessiva, cansaço e preocupação intensa, o que pode favorecer o uso indevido de substâncias psicoativas. Teixeira, ${ }^{(15)}$ refletindo sobre a vinculação entre depressão e trabalho, relatou que o "alcoolismo é a consequência mais frequente que surge da depressão, pela sobrecarga de responsabilidade e tensão no meio laboral". De acordo com a autora, o uso abusivo do álcool entre os traba- 
Ihadores pode levar ao agravamento do quadro depressivo, produzindo alterações no modo de ver o mundo e sentir a realidade.

Os Transtornos Mentais Comuns (TMC) e sua desordem mais conhecida, a depressão, foram estudados em trabalhadores do corte da cana-de-açúcar por Faria et al,(16) que avaliaram as associações entre características do trabalho rural e a ocorrência de morbidade psiquiátrica menor (MPM) entre agricultores da Serra Gaúcha e relataram uma prevalência de 38\% (IC 95\%: 34,9-40,2) para prováveis casos de MPM. Entre os que apresentavam problemas de tristeza e desânimo, observou-se uma prevalência de 19\% (IC 95\%: 16,8 - 21,0) para o uso de remédios psiquiátricos e de 5\% (IC 95\%: 5,2 $5,4)$ para hospitalizações por problemas psíquicos em algum momento da vida. No que se refere ao alcoolismo, outro importante indicador de saúde mental, 6\% (IC 95\%: 6,1 - 6,3) dos trabalhadores foram avaliados como "bebedores problema".

No estudo atual, a qualidade de vida referida comportou-se como fator de risco para o consumo abusivo de álcool entre os trabalhadores rurais da cana-de-açúcar. Embora não exista um consenso a respeito do conceito de qualidade de vida, a mesma pode ser entendida como sentido da vida, felicidade, bem-estar ou satisfação, indicando uma construção social e cultural importante e apresentando uma organização complexa, diferindo entre as pessoas de acordo com seu ambiente ou contexto, e mesmo entre duas pessoas inseridas em um ambiente similar. ${ }^{(17,18)} \mathrm{O}$ alcoolismo entre os trabaIhadores rurais é uma resposta ao estresse laboral crônico que aparece quando falham as estratégias de enfrentamento que o indivíduo, habitualmente, emprega para manejar os estressores laborais. Comportando-se como uma variável mediadora entre o estresse percebido e suas consequências, o alcoolismo é um hábito tipicamente manifestado por meio de sintomas psicológicos estando, estreitamente, relacionado com insatisfações no trabalho. Destaca-se, ainda, a dimensão social desta condição, correspondendo ao tipo de atividade la- boral do indivíduo, sua relação com aqueles com quem tem que trabalhar no seu dia-a-dia. $(5,6,7)$

Faker e Guimarães, ${ }^{(18)}$ avaliaram a saúde mental e qualidade de vida entre 217 trabalhadores dos canaviais do Mato Grosso do Sul e relataram melhor desempenho dos indivíduos que obtiveram 71,6 pontos no Componente Físico e o pior entre aqueles com 65,6 pontos no Componente Mental, de acordo com o SF-36. Tais componentes permitem a visualização dos resultados alcançados, os quais podem estar relacionados a distintas patologias: o Componente Físico, compreendendo a capacidade funcional, a dor e o estado geral dos trabalhadores, e o Componente Mental, compreendendo os aspectos emocionais, sociais e relacionados à vitalidade. As autoras ainda indicaram que o consumo abusivo do álcool entre os canavieiros esteve associado à dor, ao estado geral de saúde, à vitalidade, bem como aos aspectos sociais e de saúde mental, sugerindo que o consumo desta substância pode contribuir para a necessidade de aliviar o estado de dor física e psíquica na busca da produtividade e de maiores salários.

Em avaliação da qualidade de vida relacionada à saúde de 44 cortadores de cana-de-açúcar de uma usina sucroalcooleira no oeste do estado de São Paulo, nos períodos de entressafra e safra, CarvaIho Júnior, ${ }^{(19)}$ descreveram que a função extenuante destes trabalhadores, associada à exposição ambiental, juntamente com os hábitos específicos dessa população, como má alimentação, tabagismo e alcoolismo, podem acarretar prejuízos para a saúde física e mental. Neste estudo, observou-se que os trabalhadores que permaneceram na safra eram os que apresentavam piores aspectos sociais, sugerindo a necessidade de promoção de políticas assistencialistas de saúde.

Os resultados de Alves, ${ }^{(20)}$ corroboram os de Rocha, ${ }^{(21)}$ que analisaram a qualidade de trabalho e de vida de trabalhadores envolvidos no corte manual e mecanizado da cana-de-açúcar. Os autores constataram que os canavieiros estavam expostos a inúmeras situações de risco à saúde, predominantemente a riscos de ocorrência de problemas res- 
piratórios, osteomusculares e psicológicos. Neste estudo, $74,4 \%$ dos cortadores manuais da cana relataram fadiga e dores no corpo ao final de um dia de trabalho, enquanto $17,9 \%$ admitiram ter fadiga mental. Metade dos operadores mecânicos admitiu sentir fadiga corpórea ao final do turno, enquanto $31,2 \%$ afirmaram que sentiam fadiga mental, estresse ou tensão devido ao enfrentamento de 10 horas de trabalho por dia e descansando apenas no $12^{\circ}$ dia. Além disso, os cortadores manuais estavam sujeitos a acidentes de trabalho pela utilização do facão e picadas de animais peçonhentos, enquanto os cortadores mecânicos estavam expostos a colisões e quedas durante manutenção das máquinas. Alessi e Navarro ${ }^{(22)}$ destacaram a análise do processo de trabalho do cortador de cana-de-açúcar na região nordeste do Estado de São Paulo, revelando a exposição diária desses trabalhadores às cargas físicas, químicas e biológicas, que se traduzem em doenças, traumas, ou acidentes a elas relacionados, confirmando as análises feitas pelos autores citados anteriormente.

Consideram-se como vantagens do presente estudo a aquisição de dados primários obtidos em campo e o fato da análise ter propiciado a investigação de vários fatores de riscos simultaneamente e a multicausalidade dos eventos relacionados com a saúde dos cortadores de cana-de-açúcar. Como possíveis limitações, aponta-se que a acurácia dos dados aqui apresentados pode ter sido afetada por viés de informação, já que os mesmos foram obtidos diretamente do informante principal e se relacionavam com hábitos de vida socialmente não aceitos. Ressalta-se também que a coleta de dados foi realizada em um único espaço geográfico, podendo contribuir para a não representatividade da amostra, considerando que foram entrevistados os trabalhadores de apenas uma usina sucroalcooleira.

\section{CONCLUSÃO}

A prevalência de consumo abusivo de álcool entre os canavieiros é elevada e os principais fatores associados ao consumo são a insatisfação com o trabalho, o sentimento de tristeza ao final da jornada de trabalho e a baixa qualidade de vida.

Recomenda-se uma maior vigilância e fiscalização mais efetiva na condução do processo de trabalho nos canaviais, considerando-se as irregularidades observadas durante a coleta de dados e o grau de sofrimento identificado no presente estudo. Novos estudos com delineamento longitudinal explorando causalidade entre as variáveis envolvidas devem ser estimulados.

\section{REFERÊNCIAS}

1. Lopes M. Uso de álcool, estresse no trabalho e fatores associados entre servidores técnicoadministrativos de uma universidade pública [dissertação]. Ribeirão Preto, SP: Universidade de São Paulo; 2011.

2. Ministério da Saúde (BR). Doenças relacionadas ao trabalho: manual de procedimentos para os Serviços de Saúde. 2001 [acesso 2014 ago 12]. Disponível em: http://bvsms.saude.gov.br/bvs/ publicacoes/doencas_relacionadas_trabalhol. pdf

3. Magallón TJC, Robazzi MLCC. Consumo de álcool em trabalhadores de uma indústria em Monterrey, México. Rev Latinoam Enferm. 2005;13(n. spe):819-826.

4. Babor TF, Higgings-Biddle JC, Sauders JB, Monteiro MG. AUDIT: teste para identificação de problemas relacionados ao uso de álcool: roteiro para uso em atenção primária. Tradução de Clarissa Mendonça Corradi. Ribeirão Preto: PAI-PAD; 2003.

5. André LM. Heróis da lama: sobrecarga emocional e estratégias defensivas no trabalho de limpeza pública [tese]. São Paulo: Faculdade de Saúde Pública; 1994.

6. Silva FPP. Burnout: um desafio à saúde do trabalhador. Rev Psicol Soc Inst. 2000;2(1):5-18.

7. Moraes GTBM, Pilatti LA. Alcoolismo e as organizações: por que investir em programas de prevenção e recuperação de dependentes químicos. In: Anais do XXIV Encontro Nacional De Engenharia De Produção. 
2004. Florianópolis. [acesso 2014 jul 17].

Disponível em: www.abepro.org.br/biblioteca/

ENEGEP2OO4_EnegepO4O4_1055.pdf

8. Gonçalves JRL, Vila Luís MA. O uso de bebidas alcoólicas entre os cortadores de cana de uma agroindústria sucroalcooleira [dissertação]. Ribeirão Preto: Escola de Enfermagem; 2000.

9. 9. National Institute on Alcohol Abuse and Alcoholism. Alcohol Use Disorders Identification Test (AUDIT). 2000 [acesso 2014 June 15]. Disponivel em: http://www.niaaa.nih.gov/ NR/rdonlyres/287137A9-62BF-4EDE-A7524A351C57AOB8/O/Audit.pdf

10. 1O. Amato TC, Silveira OS, Oliveira JS, Ronzani TM. Uso de bebida alcoólica, religião e outras características sociodemográficas em pacientes da Atenção Primária à Saúde - Juiz de Fora, MG, Brasil. SMAD, Rev. eletrônica saúde mental alcool drog. 2008;4(2). [acesso 2014 jun 17]. Disponível em: http://www2.eerp.usp.br/resmad/ artigos.asp

11. 11. Ferreira LN, Bispo Júnior PJ, Sales ZN, Casotti CA, Braga Júnior AC. Prevalência e fatores associados ao consumo abusivo e à dependência de álcool. Ciênc Saúde Coletiva 2013;18(11):3409-3418. DOI: 10.159O/S141381232013001100030

12. 12. Fehlberg MF, Santos I, Tomasi E. Prevalência e fatores associados a acidentes de trabalho em zona rural. Rev Saúde Pública 2001;35(3):269-75. DOI:10.159O/SOO3489102001000300009

13. 13. Omena LP, Alves CFO. Saúde do trabalhador: o alcoolismo como sintoma do sofrimento dos Policiais Militares de Alagoas [monografia]. Maceió: Universidade Estadual de Ciências da Saúde de Alagoas; 2007.

14. 14. Marqueze EC, Moreno CRC. Satisfação no trabalho: uma breve revisão. Rev Bras Saúde Ocupacional 2005; 30(112):69-79.
15. 15. Teixeira S. A depressão no meio ambiente do trabalho e sua caracterização como doença do trabalho. Rev. Trib. Reg. Trabalho 2007;46(76):27-44.

16. 16. Faria NMX, Facchini LA, Fassa ACG, Tomasi E. Estudo transversal sobre saúde mental de agricultores da Serra Gaúcha (Brasil). Rev. Saúde Pública 1999;33(4):391-4OO. DOI: 10.1590/S0034-89101999000400011

17. 17. Pereira EF, Teixeira CS, Lopes AS. Qualidade de vida de professores de educação básica do município de Florianópolis, SC, Brasil. Ciênc Saúde Coletiva. 2013;18(7):1963-1970. DOI: 10.1590/S1413-81232013000700011

18. 18. Faker JN, Guimarães LAM. A cana nossa de cada dia: saúde mental e qualidade de vida em trabalhadores rurais de uma usina de álcool e açúcar de Mato Grosso do Sul [dissertação]. Campo Grande: Universidade Católica Dom Bosco; 2009.

19. 19. Carvalho Junior LCS, Ramos EMC, Toledo $A C$, Ceccato ADF, Macchione M, Braga ALF, et al. Avaliação da qualidade de vida relacionada à saúde de cortadores de cana-de-açúcar nos períodos de entressafra e safra. Rev. Saúde Pública. 2012;46(6):1058-65. DOI: 10.159O/ S0034-89102012000600016

20. 2O. Alves F. Por que morrem os cortadores de cana? Saúde e Soc. 2006;15(3):90-98. DOI: 10.1590/SO104-12902006000300008

21. 21. Rocha FLR, Marziale MHP, Hong OS. Work and health conditions of sugar cane workers in Brazil. Rev Esc Enferm USP. 2010;44(4):974-9.

22. 22. Alessi NP, Navarro VL. Saúde e trabalho rural: o caso dos trabalhadores da cultura canavieira na região de Ribeirão Preto, São Paulo, Brasil. Cad. Saúde Pública. 1997; 13(2):111-121. DOI: 10.1590/SO1O2$311 \times 1997000600010$ 